University of Nebraska - Lincoln

DigitalCommons@University of Nebraska - Lincoln

6-14-1999

\title{
dc Field-Induced, Phase and Polarization Control of Interference between One- and Two-Photon Ionization Amplitudes
}

\author{
N. L. Manakov \\ Voronezh State University, manakov@phys.vsu.ru \\ V.D. Osiannikov \\ Voronezh State University, Voronezh, Russia \\ Anthony F. Starace \\ University of Nebraska-Lincoln, astarace1@unl.edu
}

Follow this and additional works at: https://digitalcommons.unl.edu/physicsstarace

Part of the Physics Commons

Manakov, N. L.; Osiannikov, V.D.; and Starace, Anthony F., "dc Field-Induced, Phase and Polarization Control of Interference between One- and Two-Photon Ionization Amplitudes" (1999). Anthony F. Starace Publications. 70.

https://digitalcommons.unl.edu/physicsstarace/70

This Article is brought to you for free and open access by the Research Papers in Physics and Astronomy at DigitalCommons@University of Nebraska - Lincoln. It has been accepted for inclusion in Anthony F. Starace Publications by an authorized administrator of DigitalCommons@University of Nebraska - Lincoln. 


\title{
dc Field-Induced, Phase and Polarization Control of Interference between One- and Two-Photon Ionization Amplitudes
}

\author{
N.L. Manakov, ${ }^{1}$ V.D. Ovsiannikov ${ }^{1}$ and Anthony F. Starace ${ }^{2}$ \\ ${ }^{1}$ Physics Department, Voronezh State University, Voronezh, Russia, 394693 \\ ${ }^{2}$ Department of Physics and Astronomy, The University of Nebraska, Lincoln, Nebraska 68588-0111
}

(Received 12 January 1999)

\begin{abstract}
We demonstrate that a weak, static electric field enables nearly complete coherent phase control of the total ionization yield in a two-color ionization process using fundamental and secondharmonic radiation. The static electric field induces a dipole-forbidden resonance in the twophoton transition amplitude so that the final photoelectron states are identical to those in a single photon transition. We demonstrate also a phase controllable circular dichroism effect in the total photoelectron yield. Experimental realization of this process using alkali metal atoms is discussed. [S0031-9007(99)09357-6]
\end{abstract}

PACS numbers: 32.80.Fb, 32.80.Qk, 32.80.Rm

Coherent control of atomic and molecular processes has blossomed in the past decade, with two major approaches: one focused on manipulating the interference between alternative transition amplitudes generated by a two-color laser field [1], and the other on manipulating electronic or vibronic wave packets produced and controlled by short laser pulses [2]. The two-color approach encompasses a variety of pairs of interfering amplitudes, which differ in the kinds of transitions and in the extent of possible control. One kind is interference between a dipole-allowed three-photon transition and a one-photon, third-harmonic transition [3]. As some of the allowed final states for the two transitions are the same, control of at least some of the total ionization yield is possible, but not $100 \%$. Also, for weak laser fields, the three-photon amplitude is small, unless enhanced by an intermediate state resonance. Another kind is interference between a two-photon transition and a one-photon, second-harmonic transition [4]. For electric dipole transitions only the angular distribution of photoionized electrons can be coherently controlled: as the final states have different parity, there is no phase control of the total ionization yield, at least for weak laser fields. [For strong fields, ponderomotive potential and other effects are important [5]; phase control of above-thresholdionization spectra using a laser and its second harmonic has been achieved [6].] This scheme has been applied also to control electron motion in semiconductors [7]. A third kind is interference between two two-photon, two-color transitions, with each enhanced by an intermediate resonance state [8]. Since the final states are identical, control of the total ionization yield is possible. However, realization of this scheme depends on the availability of suitable laser frequencies and systems having appropriate intermediate energy levels. The use of static electric fields to mix levels of opposite parity in such a scheme has recently been investigated for two interfering, dipole-quadrupole, two-photon transitions [9]. Finally, the use of laser polarization and, in particular, circular dichroism (CD) to investigate and control atomic transitions is growing [10].
Static-field-induced CD in a two-photon, dipole-forbidden bound-bound transition was recently predicted [11].

We propose here the use of a static electric field to achieve nearly complete phase control of the total ionization yield for a bound-free transition resulting from interference of a resonantly enhanced, two-photon transition and the corresponding one-photon transition induced by a second-harmonic photon. We demonstrate also circular dichroism in the total ionization yield. We examine such a process for the alkali metal atoms, for which the active electron is initially in an $s$ state, and for which the dc field induces a dipole-forbidden resonance in the two-photon transition with an intermediate $s$ state.

Specifically, consider ionization of an alkali metal atom from its ground $|n s\rangle$ state by two laser beams with frequencies $\omega_{1}=\omega$ and $\omega_{2}=2 \omega$, such that $\omega=$ $\omega_{n^{\prime} n}-\Delta$, where $\Delta$, the resonance detuning, is of the order of the width $\Gamma$ of the excited level $\left|n^{\prime} s\right\rangle$. The twophoton ionization by the field

$$
\mathbf{F}_{1}(\mathbf{r}, t)=F_{1} \operatorname{Re}\left\{\mathbf{e}_{1} \exp \left[i\left(\mathbf{k}_{1} \cdot \mathbf{r}-\omega t+\varphi_{1}\right)\right]\right\}
$$

and the single-photon ionization by the field

$$
\mathbf{F}_{2}(\mathbf{r}, t)=F_{2} \operatorname{Re}\left\{\mathbf{e}_{2} \exp \left[i\left(\mathbf{k}_{2} \cdot \mathbf{r}-2 \omega t+\varphi_{2}\right)\right]\right\}
$$

both result in electrons with the same energy, $E=2 \omega-$ $I$, where $I$ is the ionization potential. We assume ionization without core excitation, which is the usual case for optical or ultraviolet frequencies. As the photoelectrons resulting from one-photon and two-photon ionization have different angular momenta, interference between the two routes to ionization shown in Figs. 1(a) and 1(b) (for weak fields) may be observed only in the photoelectron angular distribution [4]. This interference cannot influence the total ionization yield, which is a sum of the separate one-photon and two-photon ionization yields, with the former corresponding to an $|E p\rangle$ final state and the latter to a combination of $|E s\rangle$ and $|E d\rangle$ final states.

A static field $\mathbf{F}_{0}=F_{0} \mathbf{e}_{0}$, together with the two-color radiation, induces the third-order resonant path shown 


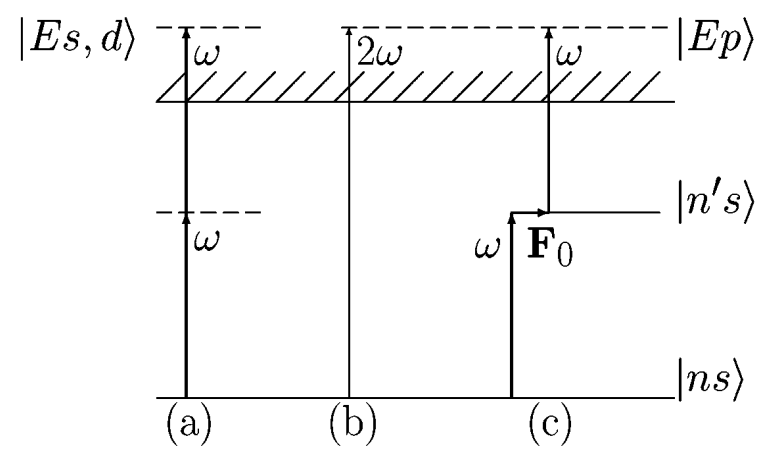

FIG. 1. Energy level schematic showing possible pathways for one- and two-photon ionization.

in Fig. 1(c). For $F_{0}$ of order $\Gamma$ in a.u. (i.e., in the range of 10 to $1000 \mathrm{~V} / \mathrm{cm}$, depending on the extent of collisional broadening of the resonant state, where typically $\Gamma=0.05-5 \mu \mathrm{eV}$ ) significant enhancement of the two-photon ionization rate occurs. In addition, the field-induced resonant amplitude interferes with that of the first-order process [cf. Fig. 1(b)]. Thus, the total ionization probability of an atom in a dc field by the laser fields in (1) and (2) has contributions from the three distinct ionization routes indicated by Figs. 1(a)-1(c), as well as from an interference between the first-order and third-order amplitudes [cf. Figs. 1(b) and 1(c)] having photoelectrons in $p$ states. Thus, the total rate is the sum of four terms,

$$
W_{\mathrm{tot}}=W_{F_{1}}^{(a)}+W_{F_{2}}^{(b)}+W_{F_{0} F_{1}}^{(c)}+W_{F_{0} F_{1} F_{2}}^{(b c)},
$$

where $a, b, c$ correspond to the processes in Fig. 1 and $b c$ refers to the interference of processes $(b)$ and $(c)$.
Every rate in the sum (3) may be written in terms of field amplitudes and radial matrix elements (RME). We designate by $R_{p}=\langle E p|r| n s\rangle$ the RME for the one-photon transition from the initial state into $p$ states of the continuum, $R_{s(d)}=\left\langle E s(d)\left|r g_{1}^{E_{n s}+\omega} r\right| n s\right\rangle$ designates that for the two-photon transition from the ground state into continuum $s$ and $d$ states, and, in the resonance approximation, the third-order RME of the dc field-induced path (c) splits into the product of $R_{n^{\prime} s}=\left\langle n^{\prime} s\left|r g_{1}^{E_{n s}+\omega} r\right| n s\right\rangle$, the RME for the second-order excitation transition into the resonant state, and $R_{p}^{\prime}=\left\langle E p|r| n^{\prime} s\right\rangle$, the first-order RME for the ionization transition from the resonant state. Here $g_{l}^{E}\left(r ; r^{\prime}\right)$ is the radial Green's function for the valence electron in a subspace of states with angular momentum $l$ (see, e.g., Ref. [12]).

The transition rates for the three ionization routes may be written in terms of the RME's as follows:

$$
\begin{gathered}
W_{F_{1}}^{(a)}=\frac{\pi F_{1}^{4}}{72}\left[l_{1}^{2} R_{s}^{2}+\frac{2}{5}\left(3-l_{1}^{2}\right) R_{d}^{2}\right] ; \\
W_{F_{2}}^{(b)}=\frac{\pi F_{2}^{2}}{6} R_{p}^{2} ; \\
W_{F_{0} F_{1}}^{(c)}=\frac{\pi F_{1}^{4} F_{0}^{2}}{54 \Gamma^{2}} \cdot \frac{\left(R_{p}^{\prime} R_{n^{\prime} s}\right)^{2}}{1+4 \Delta^{2} / \Gamma^{2}}\left|\mathbf{e}_{1} \cdot \mathbf{e}_{0}\right|^{2} .
\end{gathered}
$$

The parameter $l_{1}=\left(\mathbf{e}_{1} \cdot \mathbf{e}_{1}\right)$ in Eq. (4) designates the degree of linear polarization for the basic harmonic radiation [11]. Both phase-dependent and circular dichroism effects are due to the dc field-induced interference term, which depends on the relative phase of the two waves $\varphi=2 \varphi_{1}-\varphi_{2}$ [cf. Eqs. (1) and (2)], as follows:

$$
\begin{aligned}
W_{F_{0} F_{1} F_{2}}^{(b c)}=\frac{\pi F_{1}^{2} F_{0} F_{2}}{9 \Gamma^{2}\left(1+4 \Delta^{2} / \Gamma^{2}\right)} R_{p} R_{p}^{\prime} R_{n^{\prime} s} \cdot\left\{(2 \Delta \cos \varphi-\Gamma \sin \varphi) \operatorname{Re}\left[\left(\mathbf{e}_{1} \cdot \mathbf{e}_{0}\right)\left(\mathbf{e}_{1} \cdot \mathbf{e}_{2}^{*}\right)\right]\right. \\
\left.-(2 \Delta \sin \varphi+\Gamma \cos \varphi) \operatorname{Im}\left[\left(\mathbf{e}_{1} \cdot \mathbf{e}_{0}\right)\left(\mathbf{e}_{1} \cdot \mathbf{e}_{2}^{*}\right)\right]\right\} .
\end{aligned}
$$

Equation (7) comprises many special cases; we consider two.

Consider first linearly polarized fields, for which the term with $\operatorname{Im}\left[\left(\mathbf{e}_{1} \cdot \mathbf{e}_{0}\right)\left(\mathbf{e}_{1} \cdot \mathbf{e}_{2}^{*}\right)\right]$ in Eq. (7) disappears. For $\mathbf{e}_{1}\left\|\mathbf{e}_{2}\right\| \mathbf{e}_{0}$, we find upon substituting Eqs. (4)-(7) into Eq. (3) that the phase dependence of the total transition rate (3) is

$$
W_{\text {tot }}(\varphi)=\frac{W_{F_{2}}^{(b)}}{1+4 \Delta^{2} / \Gamma^{2}}\left[(1-f p \sin \varphi)^{2}+\left(\frac{2 \Delta}{\Gamma}+f p \cos \varphi\right)^{2}+q\left(1+\frac{4 \Delta^{2}}{\Gamma^{2}}\right)\left(\frac{F_{1}^{2}}{F_{2}}\right)^{2}\right],
$$

where

$$
f \equiv \frac{F_{0} F_{1}^{2}}{3 \Gamma F_{2}}
$$

is a field- and resonance width-dependent parameter, and

$$
p \equiv \frac{R_{p}^{\prime} R_{n^{\prime} s}}{R_{p}} \quad \text { and } \quad q \equiv \frac{1}{15}\left(\frac{R_{d}}{R_{p}}\right)^{2}\left[1+\frac{5}{4}\left(\frac{R_{s}}{R_{d}}\right)^{2}\right]
$$

are purely atomic parameters presented in terms of the RME's. Since the interference occurs only between the first- and third-order processes $(b)$ and $(c)$, corresponding effects become observable when their contribution to the total yield [given by the first two terms in square brackets in Eq. (8)] exceeds that of the second-order two-photon process $(a)$ [given by the term with factor $q$ in Eq. (8)], which provides only a phase-independent background. In terms of the dimensionless parameters,

$$
\alpha \equiv \frac{2 \Delta}{\Gamma}, \quad \beta \equiv f p, \quad \text { and } \quad \gamma \equiv q\left(\frac{F_{1}^{2}}{F_{2}}\right)^{2},
$$

we see that phase-dependent control of the total ionization yield will be maximal for $\alpha \approx \beta \approx 1$ and $\gamma \ll 1$. Numerical results for the atomic parameters $p$ and $q$ indicate that experiments with alkali metal atoms may meet these conditions rather easily (cf. Table I). Therefore we neglect $q$ or $\gamma$ in our further discussions. 
TABLE I. Natural width $\Gamma$ and parameters $p$ and $q$ for alkali metal atoms. Powers of 10 are in parentheses.

\begin{tabular}{ccccc}
\hline \hline Atom & $\begin{array}{c}\text { Resonant } \\
\text { level } n \text { 's }\end{array}$ & $\begin{array}{c}\Gamma \text { (a.u.) } \\
\text { (Ref. [15]) }\end{array}$ & $\begin{array}{c}p \text { (a.u.) } \\
\text { [cf. Eq. (10)] }\end{array}$ & $\begin{array}{c}q \text { (a.u.) } \\
\text { [cf. Eq. (10)] }\end{array}$ \\
\hline $\mathrm{Na}$ & $4 s$ & $0.65(-9)$ & $2.05(4)$ & $5.45(-1)$ \\
$\mathrm{K}$ & $5 s$ & $0.52(-9)$ & $5.26(4)$ & $1.14(-1)$ \\
$\mathrm{Rb}$ & $6 s$ & $0.52(-9)$ & $2.86(4)$ & $4.42(-2)$ \\
$\mathrm{Cs}$ & $7 s$ & $0.49(-9)$ & $3.00(4)$ & $8.36(-2)$ \\
\hline \hline
\end{tabular}

The modulation of the total ionization yield may be best expressed as the following ratio, denoted $M(\varphi)$ :

$M=\frac{W_{\text {tot }}(\varphi)-W_{\text {tot }}(\varphi-\pi)}{W_{\text {tot }}(\varphi)+W_{\text {tot }}(\varphi-\pi)}=\frac{2 \beta(\alpha \cos \varphi-\sin \varphi)}{1+\alpha^{2}+\beta^{2}}$.

For exact resonance (i.e., $\alpha=0$ ), Eq. (12) predicts $100 \%$ phase control of $M(\varphi)$ between the values $\pm M_{\max }$, where $M_{\max } \equiv 2 \beta /\left(1+\beta^{2}\right)$, as the phase varies over the range $-\pi / 2 \leq \varphi \leq+\pi / 2$. Figure 2 shows the dependence of $M(\varphi)$ on $\alpha$ for four values of the phase $\varphi$ for the case of $\beta=1$. One sees that significant phase control of the total ionization yield may be achieved over a rather large range of detuning from resonance.

Consider now cases for which the term with $\operatorname{Im}\left[\left(\mathbf{e}_{1}\right.\right.$. $\left.\left.\mathbf{e}_{0}\right)\left(\mathbf{e}_{1} \cdot \mathbf{e}_{2}^{*}\right)\right]$ contributes to Eq. (7); namely, those involving elliptical polarization of one or both photons. This contribution results in a circular dichroism effect for the total ionization probability: e.g., the photoelectron yield will differ for right- and left-handed circular polarization of either of the two radiations when the other is linearly polarized. If the second-harmonic field is linearly polarized perpendicular to the dc-field vector, $\mathbf{e}_{2} \perp \mathbf{e}_{0}$, then $\operatorname{Re}\left[\left(\mathbf{e}_{1}\right.\right.$. $\left.\left.\mathbf{e}_{0}\right)\left(\mathbf{e}_{1} \cdot \mathbf{e}_{2}^{*}\right)\right]$ vanishes and $\operatorname{Im}\left[\left(\mathbf{e}_{1} \cdot \mathbf{e}_{0}\right)\left(\mathbf{e}_{1} \cdot \mathbf{e}_{2}^{*}\right)\right]=\xi / 2$, where $\xi$ is the degree of circular polarization of the firstharmonic photons [11]. The degree of circular dichroism

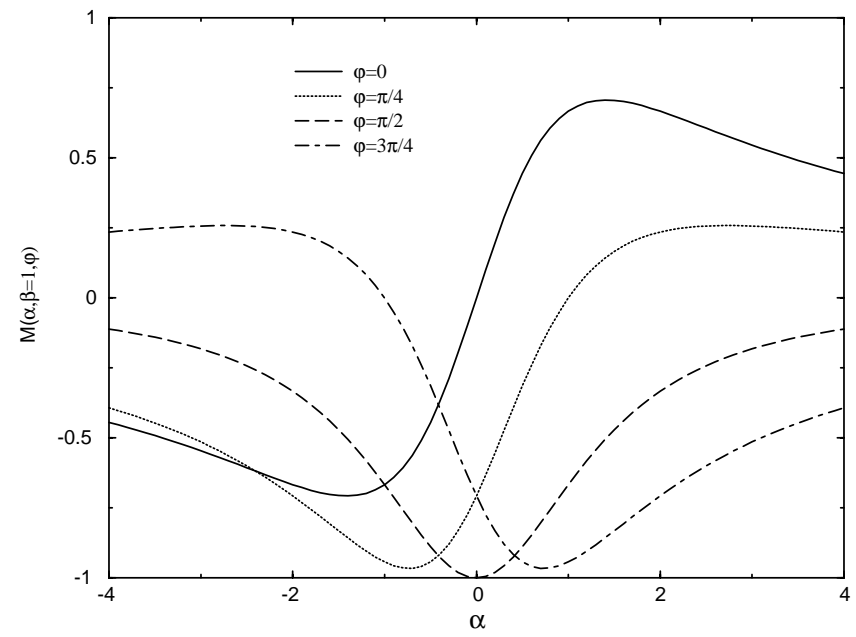

FIG. 2. Total ionization yield modulation function $M(\varphi)$ [cf. Eq. (12)] plotted vs the dimensionless detuning parameter $\alpha$ [cf. Eq. (11)] for $\beta=1$ and for four values of the relative phase $\varphi \equiv 2 \varphi_{1}-\varphi_{2}$ [cf. Eqs. (1) and (2)]. for a circularly polarized field $\mathbf{F}_{1}(\mathbf{r}, t)[\mathrm{cf}$. Eq. (1)] may be described by

$$
\begin{aligned}
D_{1} & =\frac{W_{\text {tot }}(\xi=1)-W_{\text {tot }}(\xi=-1)}{W_{\text {tot }}(\xi=1)+W_{\text {tot }}(\xi=-1)} \\
& =\frac{\beta(\alpha \sin \varphi+\cos \varphi)}{1+\alpha^{2}+\beta^{2}} .
\end{aligned}
$$

A similar measure of circular dichroism for the case of circularly polarized second-harmonic photons [cf. Eq. (2)] and linearly polarized first-harmonic photons is $D_{2}=-\sqrt{2} D_{1}$. Since $D_{1}(\varphi)=-\frac{1}{2} M(\varphi+\pi / 2)$, one sees from Fig. 2 that significant phase control of circular dichroism is also possible over a wide range of laser detunings.

Rotational invariance and symmetry arguments give a better understanding of our general result (7) and of its connection to results of others. First, the interference term (7) is asymmetric for opposite directions (i.e., $\pm \mathbf{e}_{0}$ ) of the static field $\mathbf{F}_{0}$. This asymmetry is similar to the polar asymmetry of the photoelectron yield, which is described by a term $\propto \mathbf{e}_{2} \cdot \mathbf{p}$ in the angular distribution of photoelectrons with momentum $\mathbf{p}$ [4]. In contrast with $\mathbf{F}_{0}$, however, $\mathbf{p}$ is time ( $T$ )-odd and hence the scalar product $\mathbf{e}_{2} \cdot \mathbf{p}$ must enter the angular distribution only together with another $T$-odd factor. In the situation considered in Ref. [4], i.e., interference between the paths shown in our Figs. 1(a) and $1(\mathrm{~b})$, this factor has a form $\propto \sin (\varphi+\delta)$, where $\delta$ is the difference between the continuum electron's scattering phases $\delta_{p}$ and either $\delta_{s}$ or $\delta_{d}$. Thus, the laser phase difference $\varphi$ has the same status as $\delta$; both provide phase control of the process. Obviously, neglecting the electron-core interaction, the asymmetry factor vanishes for $\varphi=0$ and has a maximum at $\varphi=\pi / 2$ (cf. Ref. [13]). In Eq. (7) [see also Eqs. (12) and (13)] the factors $\sin \varphi$ and $\cos \varphi$ enter in opposite ways in the factors multiplying $\operatorname{Re} I$ and $\operatorname{Im} I$, where $I \equiv\left(\mathbf{e}_{1} \cdot \mathbf{e}_{0}\right)\left(\mathbf{e}_{1} \cdot \mathbf{e}_{2}^{*}\right)$. This is easily explained, since the combination of vectors $\operatorname{Re} I$ is $T$-even and, therefore, the $T$-odd factor $\sin \varphi$ appears multiplied by the level width $\Gamma$, which is evidently $T$-odd also. For $\varphi=0$ the term with $\operatorname{Re} I$ is proportional to $\Delta$ and vanishes for exact resonance. On the contrary, the dichroic term, $\operatorname{Im} I$, is $T$-odd and the appropriate coefficients before it are proportional to either $\sin \varphi$ or $\Gamma$. Therefore, for $\varphi=0$ or at exact resonance circular dichroism is completely dissipation induced, which is similar to phase-independent, circular dichroism effects in (one- or multiphoton) photoprocesses with initially unpolarized targets [14]. When $\varphi \neq 0$, the phase-dependent and polarization effects in the presence of a static field lead to a variety of possible behaviors, providing great flexibility for experiments with targets having different atomic parameters. Noteworthy is the fact that a static field provides a one-photon resonance in the laseratom interaction between two $|s\rangle$ levels, which is strongly forbidden for $\mathbf{F}_{0}=0$. For this case circular dichroism formally is similar to possible parity-violating effects, caused by neutral currents, etc. [15], which lead to an admixture of $|p\rangle$ levels to the resonant $\left|n^{\prime} s\right\rangle$ level. 
Numerical data for the atomic parameters $p$ and $q$ for the alkali metal atoms, calculated according to the Fues model potential method [12], are given in Table I, together with the widths $\Gamma$ of the lowest resonant $s$ levels [16]. The large absolute values of the parameter $p$ enable the experimental observation of the effects predicted here with achievable values of the relevant field amplitudes. For example, taking $F_{0}=3 \Gamma$ and requiring the dimensionless parameter $\beta=f p$ to be unity, we determine the ratio between the amplitudes of the ac fields as $F_{1}^{2} \approx\left(10^{-5}-10^{-4}\right) F_{2}$ for the values of $p$ typical of alkali metal atoms (cf. Table I). This relation means that if the second-harmonic field is fixed at, e.g., $F_{2}=$ $10 \mathrm{kV} / \mathrm{cm}$ then the first-harmonic field varies in the range of $F_{1}=20-70 \mathrm{kV} / \mathrm{cm}$. (Note that $F_{0} \sim 3-15 \mathrm{~V} / \mathrm{cm}$ will suffice if only the radiation width accounts for the dissipation of atoms from the resonant state, while one needs $F_{0} \sim 100-1000 \mathrm{~V} / \mathrm{cm}$ if $\Gamma$ accounts also for collisional broadening under conditions typical for alkali metal atomic vapors.) The contribution of the interference term (7) may also increase when the dc field $F_{0}$ is increased instead of $F_{1}$, so that the phase-dependent interference effects may appear for lower values of $F_{1}$. As one may verify from the $q$ values in Table I, the relative contribution of the dc field-independent twophoton ionization process shown in Fig. 1(a) under the above-discussed conditions does not exceed $10^{-8}$ in any of the transitions presented in Table I.

In conclusion, we have presented a detailed analysis of a means to achieve nearly complete phase control of the total ionization yield for a bound-free transition resulting from interference of a resonantly enhanced two-photon transition and the corresponding one-photon, second-harmonic transition. A static electric field is employed to ensure that the final states reached by either of the two interfering paths are identical. This scheme also permits a CD effect for the total ionization yield. Our results are presented in the form of simple formulas for the modulation of the total ionization yield and of the CD effect as functions of the relative phase of the two laser fields. These formulas involve dimensionless parameters dependent on both atomic data and on the various laser and static field variables. For the alkali metal atoms, we have provided the relevant atomic data so that experimentalists need only to select the field variables in order to estimate the outcomes of particular measurements. We comment finally on some possible concerns regarding our treatment. First, the detuning from resonance is not so large that other intermediate resonances (e.g., $n^{\prime} p$ ) need to be considered. The $n^{\prime} s$ levels in the alkalis have large quantum defects, which keeps them well-separated from $n^{\prime} l$ levels. Second, since $n^{\prime} s$ is populated by a dipoleforbidden process, saturation of this level is not an issue. Indeed, the ratio of the decay rate of $n^{\prime} s$ (due to ionization by $F_{1}$ ) to the Rabi frequency (coupling $n^{\prime} s$ to the initial state) is proportional to $F_{1} / F_{0} \gg 1$. Hence for reasonable values of $F_{1}$ and $F_{0}$, one has ionization of $n^{\prime} s$ before Rabi oscillation occurs. This is true for any detuning, including $\Delta=0$. Hence our formulation should be generally useful to experimentalists in planning experiments for coherent control of total ionization yields with nearly complete phase control, for exploring circular dichroism effects on total ionization yields, and for possible applications in semiconductors [7].

This research was supported in part by U.S. NSF Grant No. PHY-9722110 and by Russian FBR Grants No. 9702-16407 and No. 98-02-16111. We thank P. B. Corkum, T.F. Gallagher, H. A. Rabitz, and B. W. Shore for helpful discussions.

[1] M. Shapiro, J. W. Hepburn, and P. Brumer, Chem. Phys. Lett. 149, 451 (1988); P. Brumer and M. Shapiro, Annu. Rev. Phys. Chem. 43, 257 (1992).

[2] D. J. Tanner and S. A. Rice, Adv. Chem. Phys. 70, 441 (1988); W. S. Warren, H. Rabitz, and M. Dahleh, Science 259, 1581 (1993); B. Kohler et al., Acc. Chem. Res. 28, 133 (1995).

[3] C. Chen, Y. Y. Yin, and D. S. Elliott, Phys. Rev. Lett. 64, 507 (1990); V.D. Kleiman et al., J. Chem. Phys. 102, 5863 (1995); S. Cavalieri, R. Eramo, and L. Fini, Phys. Rev. A 55, 2941 (1997).

[4] N. B. Baranova and B. Ya. Zel'dovich, J. Opt. Soc. Am. B 8, 27 (1991); N. B. Baranova et al., Sov. Phys. JETP 71, 1043 (1990); D. Z. Anderson et al., Sov. Phys. JETP 75, 210 (1992); N. B. Baranova et al., JETP Lett. 55, 439 (1992); Y. Y. Yin et al., Phys. Rev. Lett. 69, 2353 (1992); Y. Y. Yin et al., Chem. Phys. Lett. 241, 591 (1995).

[5] K. J. Schafer and K. C. Kulander, Phys. Rev. A 45, 8026 (1992); L. Gao et al., Phys. Rev. A 58, 3807 (1998).

[6] D. W. Schumacher et al., Phys. Rev. Lett. 73, 1344 (1994); D. W. Schumacher and P. H. Bucksbaum, Phys. Rev. A 54, 4271 (1996).

[7] E. Dupont et al., Phys. Rev. Lett. 74, 3596 (1995); A. Haché et al., ibid. 78, 306 (1997); W. Pötz, ibid. 79, 3262 (1997).

[8] S. T. Pratt, J. Chem. Phys. 104, 5776 (1996); F. Wang, C. Chen, and D. S. Elliott, Phys. Rev. Lett. 77, 2416 (1996); F. Wang and D. S. Elliott, Phys. Rev. A 56, 3065 (1997).

[9] B. Dobrydnev and M. Havey, Phys. Rev. A 54, 4327 (1996).

[10] J. Viefhaus et al., Phys. Rev. Lett. 77, 3975 (1996); V. Mergel et al., Phys. Rev. Lett. 80, 5301 (1998).

[11] N. L. Manakov and V.D. Ovsiannikov, J. Phys. B 30, 2109 (1997).

[12] N. L. Manakov, V.D. Ovsiannikov, and L.P. Rapoport, Phys. Rep. 141, 319 (1986).

[13] M. Yu. Kuchiev and V. N. Ostrovsky, J. Phys. B 31, 2525 (1998).

[14] N.L. Manakov, in Super-Intense Laser-Atom Physics $I V$, edited by H. G. Muller and M. V. Fedorov, NATO Advanced Study Institute, Ser. 3, Vol. 313 (Kluwer, Dordrecht, 1996), p. 153.

[15] I. B. Khriplovich, Parity Nonconservation in Atomic Phenomena (Nauka, Moscow, 1981).

[16] A. A. Radtsig and B. M. Smirnov, Parameters of Atoms and Atomic Ions (Energoatomizdat, Moscow, 1986). 